\title{
(2) OPEN ACCESS \\ Estimation of impact of RPE65-mediated inherited retinal disease on quality of life and the potential benefits of gene therapy
}

\author{
Andrew Lloyd, ${ }^{1}$ Natalia Piglowska, ${ }^{1}$ Thomas Ciulla, ${ }^{2}$ Sarah Pitluck, ${ }^{3}$ Scott Johnson, ${ }^{4}$ \\ Marric Buessing, ${ }^{4}$ Thomas O'Connell ${ }^{4}$
}

\begin{abstract}
${ }^{1}$ Acaster Lloyd Consulting
London, UK

${ }^{2}$ Clearside Biomedical (previously Spark Therapeutics) Alpharetta, Georgia, USA ${ }^{3}$ Spark Therapeutics, Philadelphia, Pennsylvania, USA ${ }^{4}$ Medicus Economics, Boston, Massachusetts, USA
\end{abstract}

\section{Correspondence to}

Dr Andrew Lloyd, Acaster Lloyd Consulting, London W2 6LG, UK; andrew.lloyd@acasterlloyd. com

Received 16 August 2018 Revised 20 December 2018 Accepted 20 December 2018 Published Online First 18 January 2019

\section{Check for updates}

(C) Author(s) (or their employer(s)) 2019. Re-use permitted under CC BY-NC. No commercial re-use. See rights and permissions. Published by BMJ.

To cite: Lloyd $A$

Piglowska N, Ciulla T, et al.

$\mathrm{Br} J$ Ophthalmol

2019:103:1610-1614.

\begin{abstract}
Background/aims In rare diseases, health-related quality of life (HRQL) data can be difficult to capture. Given the ultrarare nature of RPE65-mediated inherited retinal disease (IRD), it was not feasible to recruit a patient sample and collect HRQL data prospectively. The objectives of this study were to develop health state descriptions of RPE65-mediated IRD, and to estimate associated patient utilities.

Methods Vignette descriptions of IRD states were developed and then assessed to elicit utilities. The vignettes ranged from moderate vision loss through to hand motion to no light perception (NLP). Six retina specialists with additional expertise in IRDs provided a proxy valuation of the vignettes using generic measures of health-the 5-level version of EQ-5D-5L and Health Utility Index 3 (HUI3). The data were then scored using standard methods for each instrument.
\end{abstract}

Results Weights from both HRQL measures revealed a large decline in scores with vision loss. The EQ-5D-5L weights ranged from 0.709 for moderate vision loss to 0.152 for hand motion to NLP. The HUI3 weights ranged from 0.519 to -0.039 , respectively. A decline was seen on both measures, and the degree of decline from moderate vision loss to NLP was identical on both $(-0.56)$

Conclusion This is the first study to report HRQL weights (or utilities) for health states describing different levels of vision loss in patients with IRD, specifically those with RPE65-mediated disease. The parallel decline in scores from the EQ-5D and HUI3 corroborates the substantial impact of progressive vision loss on HRQL.

\section{INTRODUCTION}

Inherited retinal diseases (IRD) are an important cause of childhood blindness,. ${ }^{12}$ IRDs can be caused by mutations in numerous genes, including a recessive mutation in the RPE65 gene which codes for a critical enzyme in the visual cycle. Individuals with RPE65-mediated IRDs are visually impaired at low levels of lighting from infancy and the majority become fully blind in adulthood. ${ }^{34}$ Recent research has indicated that certain forms of RPE65-mediated IRDs are amenable to a gene therapy. ${ }^{5}$ On 19 December 2017, voretigene neparvovec-rzyl (LUXTURNA) was the first such gene therapy to receive approval from the US Food and Drug Administration (FDA) for the treatment of patients with confirmed biallelic RPE65 mutation-associated retinal dystrophy and viable retinal cells.
Many countries like the UK conduct reviews of the cost-effectiveness of treatments to determine if their associated health benefits represent good value relative to their cost to the health service. In cost-effectiveness analysis (CEA), the benefits of interventions are generally considered in terms of their impact on quality-adjusted life-years, reflecting length of life and health-related quality of life (HRQL). ${ }^{6}$ Consequently, HRQL estimates must be available to characterise patients with varying levels of severity in a disease under consideration in CEA. The Institute for Clinical and Economic Review (ICER) in the $\mathrm{USA}^{7}$ recently completed a CEA of LUXTURNA. However, no HRQL data were collected in the LUXTURNA clinical trials which could support CEA, and so the ICER review team was forced to rely on HRQL studies of patients with very different ocular disorders including retinal detachment, age-related macular degeneration, diabetic retinopathy, cataract, glaucoma, endophthalmitis and central retinal vein obstruction, to proxy for the effect of vision loss in patients with RPE65-mediated IRD. ${ }^{8}{ }^{9}$ The accuracy of such data in characterising HRQL in patients with RPE65-mediated IRD is limited because the pathology of these diseases and the age of participants are significantly different from those with RPE65 mutation-associated IRD. Quality of life weights (or utilities) are an essential component of a CEA because they are a measure of health benefit. No published health utilities exist for RPE65-mediated IRD.

Retinitis pigmentosa accounts for approximately half of IRDs and affects an estimated 20-30 people per $100000 .^{10}$ Leber congenital amaurosis (LCA) is less common, affecting two to three people per $100000 .^{11}$ Mutations in the RPE65 gene account for $2 \%$ of retinitis pigmentosa and $8 \%-16 \%$ of LCA diagnoses. ${ }^{11-16}$ It is believed that there are between 1000 and 2000 patients in the USA with biallelic RPE65 mutation-associated IRD. ${ }^{17}$ Given the rarity of RPE65-mediated IRD, it was not feasible to collect HRQL data prospectively, particularly given the need for estimates across all levels of disease severity. Therefore, we have used structured elicitation of HRQL data from clinical experts based on a series of case study vignettes, a method used previously for CEAs in other rare diseases. ${ }^{18} 19$ Each vignette was assessed using two generic measures of HRQL which can be used to estimate utilities needed for CEA.

\section{MATERIALS AND METHODS}

First, health state levels were defined to describe different levels of visual function in people with 
RPE65-mediated vision loss, ranging from moderate vision loss through to hand motion (HM) to no light perception (NLP). Second, health state vignettes were developed through a process that combined information gathered from background materials, feedback from an expert advisory board meeting, and interviews with clinical specialists, patients and caregivers. Finally, these vignette descriptions of IRD states were assessed to elicit utilities. Specifically, six retina specialists with additional expertise in IRDs provided a proxy valuation of the vignettes using generic measures of health-the 5-level version of EQ-5D-5L and Health Utility Index 3 (HUI3).

\section{Health state levels}

Health state descriptions were based off the American Medical Association guidelines on visual disability. For visual field (VF), categories were defined by VF radius. This was translated into sum total degrees by assuming a concentric VF and multiplying the radius by 24 . The health state descriptions were designed to describe different levels of visual function in people with RPE65-mediated vision loss:

- Moderate visual impairment, defined as either visual acuity (VA) better than 20/200 (6/60) or Goldman VF sum total degrees $>240$ (ie, radius $>10^{\circ}$ ).

- Severe visual impairment, defined as either VA from 20/200 to $20 / 500$ or Goldman VF sum total degrees $>144$ and $\leq 240$ (ie, radius $6^{\circ}-10^{\circ}$ ).

- Profound visual impairment, defined as either VA from $20 / 500$ to $20 / 1250$ or Goldman VF sum total degrees $>44$ and $\leq 144$ (ie, radius $2^{\circ}-6^{\circ}$ ).

- Counting fingers, defined as either VA 20/1250 to 20/20 000 or Goldman VF sum total degrees $\leq 44$ (ie, radius $<2^{\circ}$ ).

- HM to NLP, defined as VA worse than 20/20 000.

The state 'Counting fingers' describes a patient who cannot read any letters on a vision chart at $6 \mathrm{~m}$ but is able to count fingers that are held up at a distance of $1 \mathrm{~m}$. If the patient cannot count the fingers held up in front of him/her, the investigator then tests whether the patient can see the investigator waving his/her hand. The state 'Hand motion to no light perception' includes all patients whose vision is limited to only being able to see a waving hand or worse. ${ }^{20}$

\section{Health state vignettes}

As noted above, health state vignettes were developed through a multistep process, which was supported by experts, patients and carers during the process. First, patient testimonials were obtained to understand the day-to-day burden of RPE65-mediated IRD. Next, these testimonials were used to develop an interview guide that explored the impact of visual function on health and HRQL of different levels. The interview guide was used in exploratory interviews with clinical experts, including a panel of 12 retinal specialists and six vision rehabilitation specialists. Finally, the information from these expert interviews and patient testimonials was used to develop draft health state vignettes to describe different aspects of quality of life and wellbeing for people with different levels of vision loss. These health state vignettes were assessed for content validity by patients, carers and a retinal specialist with expertise in low vision. This process is described in more detail below.

\section{Patient and caregiver testimonials}

Spark Therapeutics shared five patient and caregiver testimonials of the day-to-day burden of RPE65-mediated IRD that had been submitted by patients and caregivers to the FDA as part of the FDA Advisory Committee reviewing the Biologics Licensing Application for LUXTURNA. The testimonials were developed prior to the start of this current project. The testimonials were used to identify the different ways RPE65-mediated IRD affected patients with this rare disease, and this was used to develop the questions for the interview guide. These testimonials were reviewed for details describing the impact of living with an IRD. This information was used to support the development of an interview guide for subsequent exploratory interviews with clinical experts. The interview guide included questions that explored the impact of visual function on health and HRQL of different levels, in terms of pain, mobility problems, daily activities, worry/anxiety/depression, usual activities, social functioning, impact on work or school, and the ability to participate in sports/activities/shopping and other related activities. This guide was used in an advisory board meeting and in one-to-one interviews with clinical experts.

\section{Expert advisory board}

An advisory board including 12 retina specialists from different ophthalmology centres in the USA was held. The experts evaluated and confirmed the clinical appropriateness of the five health state definitions designed to describe different levels of visual function. The experts also discussed the interview guide that we had developed. They confirmed the clinical relevance and recommended that vision rehabilitation specialists be recruited to take part in in-depth interviews to answer the questions in the interview guide. They also recommended that retina specialists with expertise in IRDs ultimately review vignettes to rate the impact of the description on HRQL.

\section{Interviews with vision rehabilitation specialists}

Individual qualitative interviews were also conducted with six rehabilitation experts whose role involved practical therapy for adult and paediatric patients with vision loss. (None of these experts had taken part in the advisory board.) The experts were based in the USA and the UK. The experts represented professionals working at low vision clinics: a low vision specialist optometrist, a vision rehabilitation specialist, a resident in a specialist low vision clinic, an ophthalmologist, a consultant ophthalmologist and a retinal surgeon.

\section{Cognitive debrief interviews}

Using the information from the expert interviews and patient testimonials, draft health state vignette descriptions were developed. These vignettes described different aspects of quality of life and well-being for people with different levels of vision loss (see online supplementary appendix 1). A further set of interviews was conducted in order to test the content validity of the vignettes in people with IRD (or their carers). Adults with RPE65-mediated IRD and carers of children/adolescents with RPE65-mediated IRDs were recruited with assistance from a patient advocacy group (Sofia Sees Hope). In these interviews, the vignette descriptions were read to the participant one sentence at a time. The participants commented on the accuracy of the vignettes one sentence at a time. They were asked to rate the vignette that matched the respondent's current level of vision loss and also the moderate vision loss state.

Additionally, a retinal specialist with expertise in low vision assessed the accuracy of the three more advanced stages of vision loss: 'Profound', 'Counting fingers' and 'Hand motion to no light perception' using a similar cognitive debriefing approach. The final states are included in the online supplementary appendix 1. 


\section{Health state valuation}

Six retina specialists with expertise in IRDs (who had not participated in the aforementioned expert advisory board) completed interviews to provide a proxy valuation of the vignettes using generic measures of HRQL. The experts were asked to read each vignette and to draw on their clinical experience to extrapolate how patients would be affected by the severity of vision loss described in the vignette. After each vignette, the experts rated the impact of the description on HRQL by completing the EQ-5D-5 $\mathrm{L}^{21}$ and HUI3 ${ }^{22}$ questionnaires. The EQ-5D-5L data were scored using the van $\mathrm{Hout}^{23}$ scoring algorithm.

\section{RESULTS}

\section{Patient and caregiver testimonials}

As noted above, patient testimonials were obtained to understand the day-to-day burden of RPE65-mediated IRD. Participants described the loss of visual function, including VA and VF defects, night blindness and loss of peripheral vision. They also described the impact that the vision loss had on day-to-day life. For example, one child (aged 5) had become timid and very dependent because of her fear of falling or bumping into items. In another case, a mother of an older child (aged 15) described the social impact of vision loss related to a loss of eye contact and inability to pick up on social cues. This child also struggled to participate in sports and other outdoor activities. Going out to restaurants, especially in the evening, was also difficult. Similarly, the father of a young woman described the social isolation she had experienced earlier in her life, which meant that she had had very few friends.

\section{Clinician input}

Patient testimonials were used to develop an interview guide to explore the impact of visual function on health and HRQL of different levels. The interview guide was used in exploratory interviews with clinical experts, including a panel of 12 retinal specialists and six vision rehabilitation specialists. The major feedback from the retina specialist advisory board was that IRDs can cause severe and progressive loss of peripheral VF, nyctalopia and ultimately loss of central vision, all of which are associated with severe impairment in functional vision and quality of life. Some comments from the advisory board meeting were incorporated into health state descriptions in later stages; these were: patients in all health states have affected night vision/difficulty seeing at transition times (eg, sunset) and young people struggle with the idea they cannot drive.

The six vision rehabilitation specialists provided very detailed information regarding the impact of vision loss on different aspects of patients' lives which was used to develop the vignettes. They described the nature of the vision loss and how it can change over time; and the impact on everyday life and ability to complete everyday activities such as school, work or social activities. These interviews also provided some information regarding the impact of severe vision loss on patients' psychological health, relationships and family life. Rehabilitation specialists in the US and UK were recruited so that the information would reflect practice in both countries.

\section{Cognitive debrief interviews}

Information from expert interviews and patient testimonials was used to develop draft health state vignettes, which were assessed for content validity by patients, carers and a retinal specialist with expertise in low vision. Three interviews were conducted with mothers of children with vision loss ranging in age from 5 to
16 (one with severe vision loss and two with moderate) and two adults with RPE65-mediated IRD (one with profound vision loss and the other with moderate). The general feedback from these interviews was that most sections of the vignettes were accurate. Some specific areas of feedback led to changes in the vignette descriptions. The participants felt strongly that in the moderate vision loss state people did not need a cane or assistance to cross a street safely. The parents of the children with moderate vision loss indicated that it was more accurate to state that their children needed a lot of light to see (eg, a flashlight), as opposed to relying on visual aids. References to poor academic performance and motivation at school or work were deleted. One of the adult participants said that the social isolation and need for adaptations in the home was overstated. He described how he simply uses Uber and other ride-sharing services to commute and uses voice recognition devices for assistance in the home.

The clinical expert also confirmed that much of the content of the vignettes was accurate, but some recommendations for changes were made. She suggested that even in the two most severe states, patients can cross a street safely with the help of visual aids and can navigate outside with help. The expert reported that in the 'Counting fingers' state, participants cannot see during the day as well as at night. The expert also commented that in terms of psychological impact, the word 'devastating' is only appropriate for the three most severe states.

\section{Health state valuation}

Six retina specialists with expertise in IRDs completed interviews to provide a proxy valuation of the health state vignettes using generic measures of HRQL. All six retina specialists participated in the chronic care of patients with IRDs in different settings across hospitals and clinics in the USA. They had an average of 20 years of experience working with patients with IRDs (range: 4-28 years).

One clinical expert stated that he believed it was not possible to rate the emotional functioning domains on the EQ-5D-5L and HUI3 and therefore he did not provide responses for these domains. This meant that the data could not be scored as the EQ-5D-5L and HUI3 require a response to all items to produce a score. Therefore, for this participant, the missing data were replaced with the most common (or modal) response for that domain from the other experts.

The EQ-5D-5L scores ranged from 0.71 (moderate vision loss) to 0.15 (HM to NLP), with an overall range of 0.56 (table 1 and figure 1). The HUI3 scores for each state are lower than the corresponding EQ-5D-5L value. They range from 0.52 to -0.04 , with an overall range of scores of 0.56 (table 1 and figure 1). Despite the low sample size, the low SDs in table 1

$\begin{aligned} & \text { Table } 1 \text { IRD health states and HRQL scores using EQ-5D-5L and } \\
& \text { HUI3 from six clinical experts }\end{aligned}$
\begin{tabular}{llllll}
\hline & EQ-5D-5L & & & HUI3 \\
\cline { 2 - 3 } \cline { 5 - 6 } & Mean & SD & & Mean & SD \\
\hline Moderate vision loss & 0.709 & 0.094 & & 0.519 & 0.160 \\
Severe vision loss & 0.615 & 0.044 & & 0.363 & 0.112 \\
Profound vision loss & 0.515 & 0.071 & & 0.223 & 0.102 \\
Counting fingers & 0.354 & 0.063 & & 0.141 & 0.092 \\
\hline Hand motion to NLP & 0.152 & 0.114 & -0.039 & 0.069 \\
\hline
\end{tabular}

EQ-5D-5L, 5-level version of EuroQol-5 Dimension; HRQL, health-related quality of life; HUI3, Health Utility Index 3; IRD, inherited retinal disease; NLP, no light perception. 


\section{Utility estimates for IRD health states}

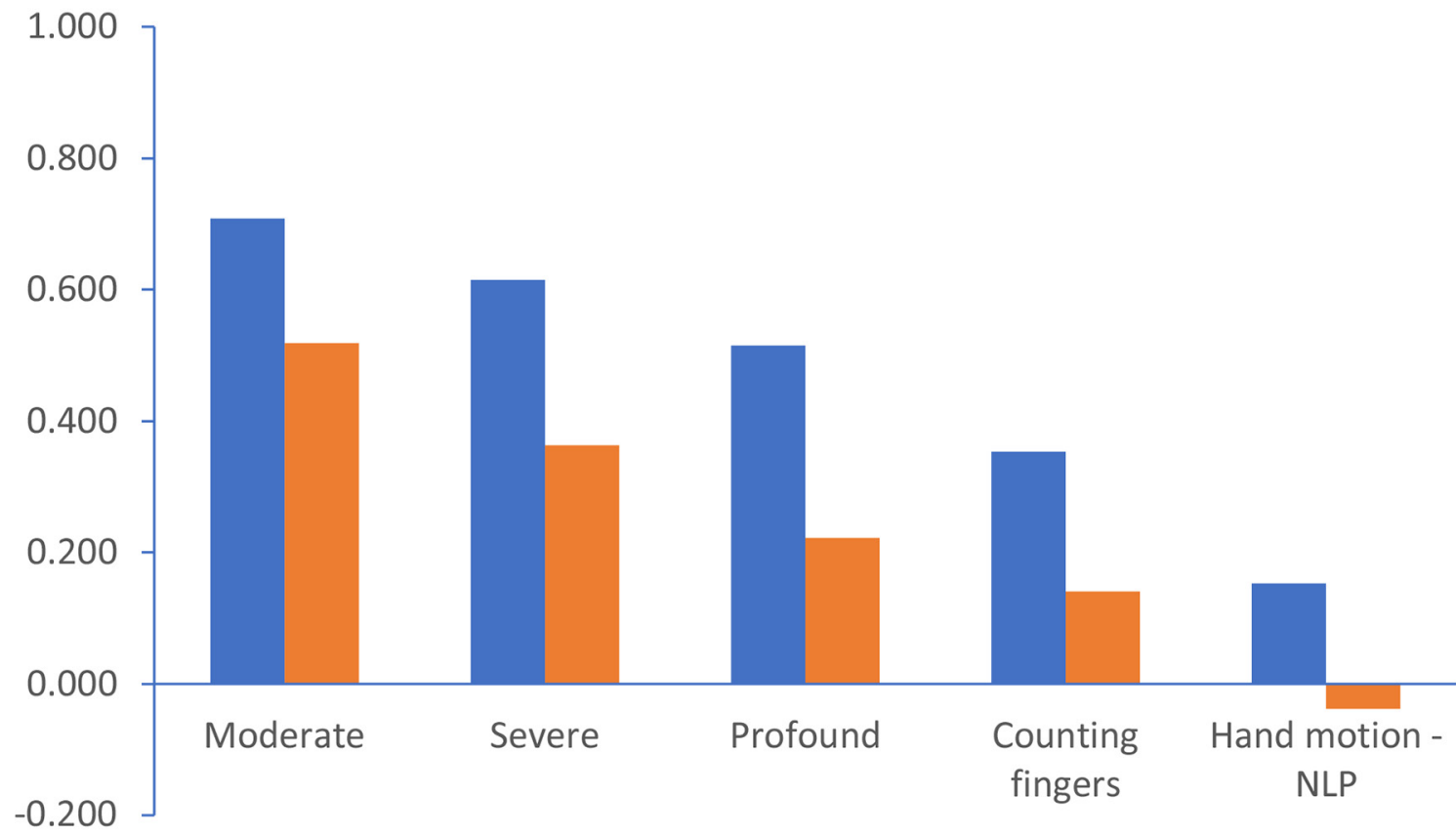

口EQ-5D-5L $\square$ HUI3

Figure 1 HRQL scores for IRD states from EQ-5D-5L and HUI-3 show the same pattern but HUI-3 scores are consistently lower. EQ-5D-5L, 5-level version of EuroQol-5 Dimension; HUI3, Health Utility Index 3; IRD, inherited retinal disease; NLP, no light perception.

indicate a high level of independent agreement between the experts in their ratings of each state.

As noted above, one clinical expert believed it was not possible to rate the emotional functioning domains on the EQ-5D-5L and HUI3 and therefore he did not provide responses for these domains. As a sensitivity analysis, the modal response used to impute the missing information was replaced with the best level (not anxious or depressed) and worst level (extremely anxious or depressed) for this participant. On the EQ-5D (which has only five dimensions and is likely the most sensitive to this imputation) the SDs for states were moderate 0.10 and 0.18 ; severe 0.06 and 0.14 ; profound 0.08 and 0.15 ; counting fingers 0.12 and 0.09 ; and HM to NLP 0.14 and 0.11 .

\section{DISCUSSION}

This study reports HRQL weights (or utilities) for health states describing different levels of vision loss in patients with RPE65-mediated IRD. These utilities were derived based on expert elicitation interviews, which used vignette descriptions of different levels of vision loss in order to elicit data from experts. The use of vignettes to capture HRQL data for economic evaluation is quite common. ${ }^{24}$ In the current study, different sources of information were used to develop five vignettes which were designed to describe health and quality of life. The health utility weights from the two HRQL measures used revealed a large decline in scores with vision loss. A decline was seen on both measures, and the degree of decline from moderate vision loss to NLP was identical on both $(-0.56)$. The HUI3 scores were lower than the EQ-5D-5L scores, which is likely due to the fact that the HUI3 includes a domain specifically related to vision. Furthermore, while the use of the EQ-5D is generally encouraged by most international health technology assessment bodies for purposes of reimbursement decision-making, the most appropriate measure for capturing HRQL may vary depending on the disease under consideration. For example, Longworth et $a l^{25}$ note, 'EQ-5D was valid and responsive for skin conditions and most cancers; in vision, its performance varied according to aetiology; and performance was poor for hearing impairments.'

The qualitative data from the patient testimonials and the interviews with vision specialists provided a rich description of the impact of having an IRD on patients' lives. Many areas of quality of life are affected by this level of vision loss including independence, mental health and the ability to engage socially with others. RPE65-mediated vision loss typically affects children and young adults, which perhaps accentuates some of the difficulties that people experience. Patients' declining vision limits their opportunities during their formative teenage and young adult years, the same period in which their peers' opportunities are expanding. The rehabilitation specialists were positive about the possibilities for improving the lives of patients with IRDs, but also were realistic about the substantial burden of very severe vision loss. The qualitative picture that informed the vignettes supports the face validity of the utility findings. The utility values are low, especially for the more severe states, and the qualitative work supported these scores.

The structured elicitation methods described above were adopted because of the difficulties in capturing new HRQL data in an extremely rare condition like RPE65-mediated IRD. It would have been very challenging to recruit samples of patients at each level of vision loss in order to capture HRQL data for the purposes of modelling. This is work that could be conducted in the future to verify these findings. In this study, the vignettes that were developed were valued by asking expert physicians to judge the impact of each state on HRQL based on their experience, and then provide a proxy rating of this using EQ-5D. The utility values were then estimated using the EQ-5D scoring weights. In the literature, it is more typical for vignettes to be rated in a time trade-off exercise with the general public. We favoured 
using clinicians here because the experience of severe vision loss may be difficult for the public to imagine. It also allowed us to describe some specific clinical information which may not be readily understood by the general public and the resulting utilities were rooted in the EQ-5D value set. The method could have been improved on by also asking people with RPE65-mediated IRD or their caregivers to also provide ratings.

Some limitations in the work should be considered when reviewing the results. The content of the vignettes drove the utility results; as such, the results are dependent on the accuracy of the vignette descriptions. For this reason, multiple rounds of interviews were conducted to develop these materials, drawing qualitative descriptions of the impact of vision loss from healthcare providers, parents, patients and carers. Moreover, in the final rounds of interviews, a consensus emerged that the vignettes were accurate, further supporting the results obtained. Valuations were provided by six retina specialists. Retina specialists with extensive expertise in IRDs represent an even smaller fraction of an already small subspecialty. The six retina specialists provided quite consistent responses to the EQ-5D-5L and HUI3 and so we believe that undertaking additional interviews would not likely have provided significantly different information. It should also be noted that one of the retinal experts was unable to judge the psychological status of people in each state and so the modal response from the other participants was imputed. The use of the modal response for this participant may have reduced the SD in the utilities slightly. The sensitivity analysis shows what the possible range of SDs could have been.

The findings are corroborated by similar values obtained using a different methodology in a different retinal disease. ${ }^{8}$ In that study, researchers derived utility weights directly from patients with macular degeneration, diabetic retinopathy or other causes of vision loss. Brown et al report values ranging from 0.26 (NLP), 0.47 (light perception to counting fingers) and 0.65 (20/200 to 20/400), similar to the current study. LUXTURNA has been reviewed by the ICER which is an independent body in the USA that evaluates the clinical and economic value of treatments (https://icer-review.org/). In their economic evaluation of LUXTURNA they relied on quality of life weights (utilities) from people with diabetic retinopathy. ${ }^{9}$ In that study, EQ-5D scores for patients with retinopathy were 0.53 (6/60 to 6/120) and 0.34 (counting fingers to $\mathrm{HM}$ ); while the equivalent values in this study were 0.62 and 0.36 to 0.15 . The higher values in the present study may reflect the younger population without type 2 diabetes.

In conclusion, this is the first study to report HRQL weights (or utilities) for health states describing different levels of vision loss in patients with RPE65-mediated IRDs. The health utility weights from the two HRQL measures revealed a large decline in scores with vision loss, corroborating the substantial impact of progressive vision loss on HRQL.

Acknowledgements We acknowledge the support and help provided by the patient advocacy groups and their members, the physicians and the rehabilitation specialists involved in this research.

Contributors AL designed the study, helped develop study materials, analysed data and wrote the paper. NP helped develop study materials and undertook some of the study interviews. SP and TC provided input into the study materials and reviewed and edited drafts of the paper. TOC, SJ and MB provided input into the study materials, reviewed the health states and reviewed and edited drafts of the paper.

Funding This work was supported by Spark Therapeutics.

Competing interests AL and NP work for Acaster Lloyd, a private consultancy which was paid a fixed fee for work on this project. SJ, MB and TOC work for Medicus Economics and were engaged by Spark Therapeutics to provide economic modelling services on the project for a fixed fee. TC and SP are employees of Spark Therapeutics, which owns LUXTURNA — a treatment for RPE65-mediated IRD.

Patient consent for publication Not required.

Provenance and peer review Not commissioned; externally peer reviewed.

Open access This is an open access article distributed in accordance with the Creative Commons Attribution Non Commercial (CC BY-NC 4.0) license, which permits others to distribute, remix, adapt, build upon this work non-commercially, and license their derivative works on different terms, provided the original work is properly cited, appropriate credit is given, any changes made indicated, and the use is non-commercial. See: http://creativecommons.org/licenses/by-nc/4.0/.

\section{REFERENCES}

1 Sharif W, Sharif Z. Leber's congenital amaurosis and the role of gene therapy in congenital retinal disorders. Int J Ophthalmol 2017;10:480-4.

2 Steinkuller PG, Du L, Gilbert C, et al. Childhood blindness. J Aapos 1999;3:26-32.

3 Kumaran N, Moore AT, Weleber RG, et al. Leber congenital amaurosis/early-onset severe retinal dystrophy: clinical features, molecular genetics and therapeutic interventions. Br J Ophthalmol 2017;101:1147-54.

4 Reape KZ, Chung DC, Schaefer G, et al. Natural history of individuals with retinal degeneration due to biallelic mutations in the Rpe65 gene. Invest. Ophthalmol. Vis. Sc 2017;58:1488.

5 Russell S, Bennett J, Wellman JA, et al. Efficacy and safety of voretigene neparvovec (AAV2-hRPE65V2) in patients with RPE65 -mediated inherited retinal dystrophy: a randomised, controlled, open-label, phase 3 trial. The Lancet 2017;390:849-60.

6 Drummond MF, Sculpher MJ, Claxton K. Methods for the economic evaluation of health care programmes. Oxford university press, 2015.

7 Institute for Clinical and Economic Review Voretigene Neparvovec. Final Report and Meeting Summary. 2018. Available: https://icer-review.org/material/voretigene-finalreport/ [Accessed 6 June 2018].

8 Brown MMet al. Utility values associated with blindness in an adult population. $\mathrm{Br} J$ Ophthalmol 2001;85:327-31.

9 Lloyd A, Nafees B, Gavriel S, et al. Health utility values associated with diabetic retinopathy. Diabet Med 2008;25:618-24.

10 Fahim A, Daiger S, Weleber R. Nonsyndromic retinitis pigmentosa overview. 2018. Available: https://www.ncbi.nlm.nih.gov/books/NBK1417/?report=printable [Accessed 14 Aug 2018].

11 Stone EM. Leber congenital Amaurosis-A model for efficient genetic testing of heterogeneous disorders: LXIV Edward Jackson memorial lecture. Am J Ophthalmol 2007; 144:791-811.

12 Simovich MJ, Miller B, Ezzeldin H, et al. Four novel mutations in the Rpe65 gene in patients with Leber congenital amaurosis. Hum Mutat 2001;18:164.

13 Wang $F$, Wang $H$, Tuan $H-F$, et al. Next generation sequencing-based molecular diagnosis of retinitis pigmentosa: identification of a novel genotype-phenotype correlation and clinical refinements. Hum Genet 2014:133:331-45.

14 Morimura H, Fishman GA, Grover SA, et al. Mutations in the Rpe65 gene in patients with autosomal recessive retinitis pigmentosa or Leber congenital amaurosis. Proceedings of the National Academy of Sciences 1998;95:3088-93.

15 Thompson D, Gyürüs P, Fleischer L. Genetics and phenotypes of RPE65 mutations in inherited retinal degeneration. 2018. Available: http://iovs.arvojournals.org/article. aspx? articleid=2162347 [Accessed 14 Aug 2018].

16 Astuti GDN, Bertelsen M, Preising MN, et al. Comprehensive Genotyping Reveals RPE65 as the most frequently mutated gene in Leber congenital amaurosis in Denmark. Eur J Hum Genet 2016:24:1071-9.

17 Population clock. 2018. Available: https://www.census.gov/popclock/ [Accessed 14 Aug 2018].

18 Pastores G, Bracken J, Hughes J. Estimation of resource use and quality of life in phenylketonuria (PKU) patients in Ireland. 13th International Congress of Inborn Errors of Metabolism 2017

19 Lloyd A, Tomazos IC, Gallop K. Effect of Asfostase alfa treatment on health states and ambulatory function among patients with hypophosphatasia. J Bone Mineral Res 2017;32:\$282.

20 Marsden J, Stevens S, Ebri A. How to measure distance visual acuity. Community Eye Health 2014;27:16

21 Herdman M, Gudex C, Lloyd A, et al. Development and preliminary testing of the new five-level version of EQ-5D (EQ-5D-5L). Quality of Life Research 2011;20:1727-36.

22 Horsman J, Furlong W, Feeny D, et al. The Health Utilities Index (HUI®): concepts, measurement properties and applications. Health and Quality of Life Outcomes 2003; $1: 54$

23 van Hout B, Janssen MF, Feng Y-S, et al. Interim scoring for the EQ-5D-5L: mapping the EQ-5D-5L to EQ-5D-3L value sets. Value in Health 2012;15:708-15.

24 Wolowacz SE, Briggs A, Belozeroff $V$, et al. Estimating health-state utility for economic models in clinical studies: an ISPOR good research practices Task Force report. Value in Health 2016;19:704-19.

25 Longworth L, Yang Y, Young T, et al. Use of generic and condition-specific measures of health-related quality of life in NICE decision-making: a systematic review, statistical modelling and survey. Health Technol Assess 2014;18:1-224. 